\title{
Percutaneous Nephrostomy
}

\author{
Brian Funaki, M.D., ${ }^{1}$ and Joshua A. Tepper, M.D. ${ }^{2}$
}

$\mathrm{P}_{\text {ercutaneous nephrostomy has been performed }}$ for 50 years and remains one of the most fundamental nonvascular interventional radiology procedures. ${ }^{1-3}$ Typically the procedure is done for urinary diversion in patients with an obstruction or a distal leak or fistula. Other indications include access for treatment of nephrolithiasis or ureteral strictures. Concomitant obstruction and sepsis is usually considered an indication for urgent nephrostomy. There are two common methods used for catheter insertion: the single-stick and double-stick techniques. I favor the former method.

\section{SINGLE-STICK TECHNIQUE FOR PERCUTANEOUS NEPHROSTOMY}

\section{Preprocedure}

In all patients, any coagulopathy should be corrected prior to procedures when possible. All patients are prepped and draped in standard fashion. We routinely administer $1 \mathrm{~g}$ ceftizoxime (Cefizox, Fujisawa, Deerfield, IL) prior to procedures.

\section{Procedure}

Using real-time ultrasound guidance, punctures are directed at a posterior calyx using a 21-gauge needle (Accustick introducer set; Boston Scientific, Natick, MA). In thin patients, real-time needle visualization is usually possible. In general, I choose the shortest path to the kidney, which is usually slightly lateral to the lumbar musculature of the flank (Fig. 1A ). It is important not to puncture too far laterally as the right or left colon may be inadvertently traversed in some patients. If possible, a subcostal approach is used although intercostal punctures are necessary in some patients. A mid- or lower-pole calyx is typically most accessible and facilitates tube placement. Parenthetically, accessing an upper-pole calyx may sometimes be necessary; if so, using an intercostal approach may be preferable to a subcostal approach particularly if the path to the kidney is sharply angled in a cephalad direction. If the access path is too steep, it is difficult to pass the tube "over the bend" into the renal pelvis as the vector of force is toward the head rather than down the ureter.

After puncture, gentle suction is placed on the needle until urine is aspirated; a small amount of contrast is injected to opacify the collecting system. It is important not to overdistend the system with contrast as this can cause sepsis or rupture a calyx. A 0.018-inch hydrophilic tipped rigid guide wire (V18 control wire; Boston Scientific) is advanced into the collecting system and if possible into the ureter (Fig. 1B). The needle is removed and coaxial 4- and 6-French dilators are inserted over the 0.018 -inch guide wire (Fig. 1C). The inner diameter of the inner 4-French dilator is designed to accept an 0.018 -inch guide wire. A rigid 0.035 -inch guide wire (Amplatz superstiff guide wire; Boston Scientific) is inserted through the 6-French dilator and the tract is serially dilated using 7- and 9-French dilators. An 8-French nephrostomy catheter (Boston Scientific) with an inner metal stiffener is then advanced over the guide wire (Fig. 1D). Once the tube reaches the calyx, it is unscrewed from the stiffener and advanced over the wire into the pelvis (Fig. 1E). The tube is affixed to the skin and left to bag drainage.

\section{Postprocedure}

Urinary output should be charted. In most patients, urine will be blood-tinged initially but should clear overnight. I wait several days prior to performing diagnostic nephrostograms if these are necessary. At that time, nephrostomy catheters can be exchanged for either ureteral stents or nephroureterostomy catheters.
${ }^{1}$ Section of Vascular and Interventional Radiology, University of Chicago Hospitals, Chicago, Illinois; ${ }^{2}$ Department of Radiology, Northwestern University, Chicago, Illinois.

Address for correspondence and reprint requests: Brian Funaki, M.D., Section of Vascular and Interventional Radiology, University of Chicago Hospitals, 5840 S. Maryland Avenue, MC 2026, Chicago, IL 60637.
Complications in Interventional Radiology; Guest Editor, Jonathan Lorenz, M.D.

Semin Intervent Radiol 2006;23:205-208. Copyright (C) 2006 by Thieme Medical Publishers, Inc., 333 Seventh Avenue, New York, NY 10001, USA. Tel: +1(212) 584-4662.

DOI 10.1055/s-2006-941451. ISSN 0739-9529. 

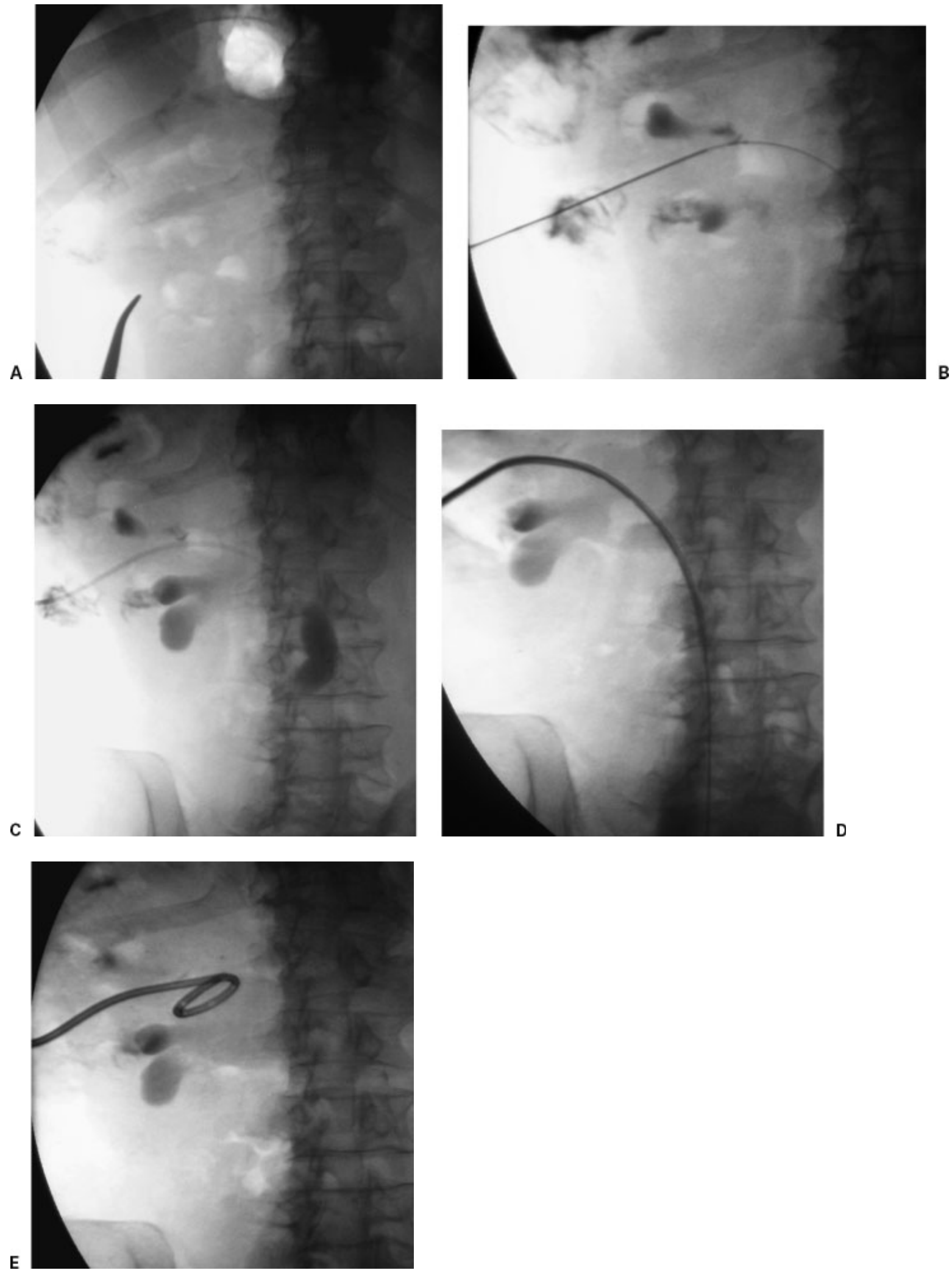

Figure 1 Percutaneous nephrostomy insertion using single-stick technique. (A) After ultrasound localization, a fluoroscopic image demonstrates overlying ribs and enables appropriate puncture site choice. (B) After ultrasound-guided 21-gauge needle puncture, a small amount of urine is aspirated and contrast is injected. Fluoroscopic image shows a 0.018-inch guide wire is advanced through the needle into the ureter. (C) The needle has been exchanged for coaxial dilators. Fluoroscopic image shows contrast injection into the ureter to confirm position. (D) The 0.018-inch guide wire has been exchanged for a rigid 0.035 -inch guide wire and the nephrostomy tube is being inserted over this wire. (E) Final fluoroscopic image shows nephrostomy coiled in renal pelvis. 
A
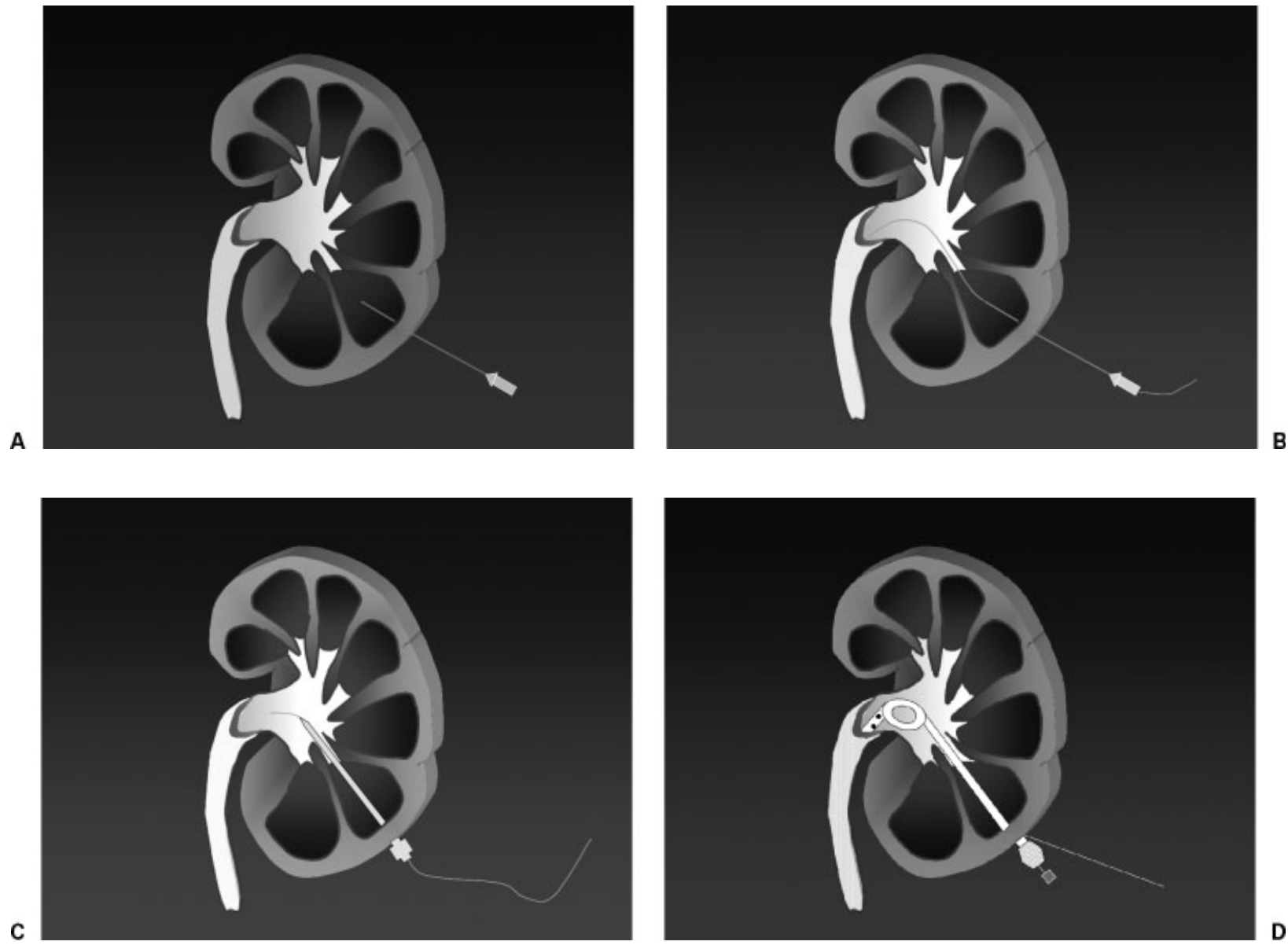

Figure 2 Illustration of single-stick nephrostomy. (A) A 21-gauge needle is directed into posterior calyx. (B) A 0.018-inch guide wire is advanced into the ureter. (C) Serial dilatation is performed over a 0.035 -inch guide wire. (D) Pigtail catheter is positioned in the renal pelvis.

\section{DISCUSSION}

Many different techniques of percutaneous nephrostomy have been described with most being a variation of either the "single-stick" (Fig. 2) or "double-stick" technique. The double-stick technique is similar to the single-stick technique except that after the collecting system is accessed, air or carbon dioxide is injected to visualize a posterior calyx, which is then punctured and used for tube insertion. Advocates of the double-stick method note that the use of two needles ensures a posterior calyceal puncture through Brodel's avascular zone, which minimizes the risk of arterial injury and urinoma formation and facilitates long-term tube function.

Advantages of single-stick nephrostomy include shorter procedure time, less radiation exposure for $\mathrm{pa}$ tients and physicians, and decreased cost. In my hospital, different interventional radiologists use one technique or the other. In a retrospective review of both methods, we found no significant differences between techniques in terms of tube function or complications. ${ }^{4}$ There are several possible explanations for these findings. I personally believe that both techniques are effective in posterior calyceal puncture and tube insertion. In terms of bleed- ing, the double-stick technique does not guarantee endon puncture of a posterior calyx because there is a wide variation in both the arterial supply to the kidney and the rotation of the kidney in the retroperitoneum. A side-on puncture could lacerate the interlobar arteries, which cross the infundibula. Puncture through Brodel's avascular zone is therefore not reliably possible in all patients. The double-stick technique has the added disadvantage of requiring a minimum two punctures through the kidney, which may increase the risk of bleeding. Given the similarity in results between the two methods and due to the fact that the single-stick method is clearly faster and cheaper, in my opinion, the single-stick method should be used predominantly for nephrostomy with one caveat. If it is difficult to visualize the kidney during ultrasound-guided puncture or if the procedure is being performed on a patient without hydronephrosis, the renal pelvis may be punctured instead of a posterior calyx. In this instance, it is advisable to switch to a double-puncture technique.

Several different types of tubes may be used for drainage. I typically insert a nephrostomy catheter at the time of initial drainage with a few exceptions. In 
demented or combative patients, a nephroureterostomy catheter is preferable as it is more difficult to displace. This requires guide wire advancement into the bladder, which may be difficult in acute ureteral obstructions. Because more resistance occurs when deploying the longer nephroureterostomy catheter (particularly if traversing a ureteral obstruction), it is helpful to place a peel-away sheath from the skin site to kidney. The tube is advanced over the stiff wire, through the sheath down the ureter, and into the bladder. Either a nephrostomy or nephroureterostomy can be converted to a ureteral stent at a later date if appropriate.

\section{REFERENCES}

1. Dyer RB, Regan JD, Kavanagh PV, Khatod EG, Chen MY, Zagoria RJ. Percutaneous nephrostomy with extensions of the technique: step by step. Radiographics 2002;22:503-525

2. Farrell TA, Hicks ME. A review of radiologically guided percutaneous nephrostomies in 303 patients. J Vasc Interv Radiol 1997;8:769-774

3. Maher MM, Fotheringham T, Lee MJ. Percutaneous nephrostomy. Semin Intervent Radiol 2000;17:329-340

4. Funaki B, Vatakencherry G. Comparison of single-stick and double-stick techniques for percutaneous nephrostomy. Cardiovasc Intervent Radiol 2004;27:35-37 